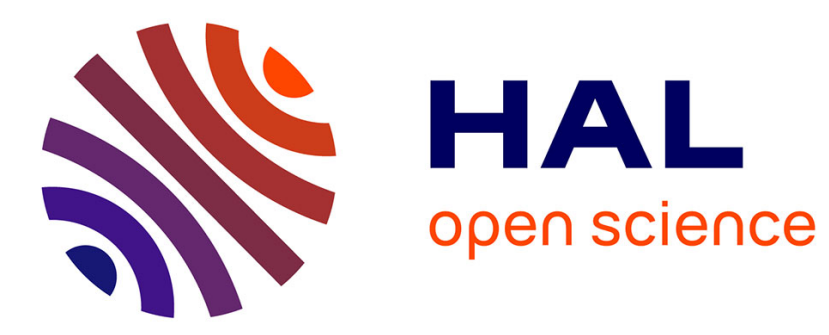

\title{
Les Langagiciels : derrière l'objet informatique, une certaine conception des apprentissages
}

\author{
Jacqueline Puyalet
}

\section{To cite this version:}

Jacqueline Puyalet. Les Langagiciels: derrière l'objet informatique, une certaine conception des apprentissages. La nouvelle revue de l'adaptation et de la scolarisation, 2008, 43, pp.89-95. hal02481664

\section{HAL Id: hal-02481664 \\ https://hal-inshea.archives-ouvertes.fr/hal-02481664}

Submitted on 17 Feb 2020

HAL is a multi-disciplinary open access archive for the deposit and dissemination of scientific research documents, whether they are published or not. The documents may come from teaching and research institutions in France or abroad, or from public or private research centers.
L'archive ouverte pluridisciplinaire HAL, est destinée au dépôt et à la diffusion de documents scientifiques de niveau recherche, publiés ou non, émanant des établissements d'enseignement et de recherche français ou étrangers, des laboratoires publics ou privés. 


\section{Les Langagiciels: derrière l'objet informatique, une certaine conception des apprentissages}

Jacqueline PUYALET

Chargée de formation

INS HEA, Suresnes

Résumé: Les Langagiciels sont un outil pédagogique, développé dans un environnement informatique, par un groupe d'enseignants, de formateurs, de chercheurs concernés par les difficultés d'apprentissage en lecture et écriture. L'article s'efforce de mettre en évidence les principales caractéristiques de l'outil et ses modalités d'utilisation. Constitué de sept programmes informatisés, ciblant chacun prioritairement un petit nombre de compétences nécessaires à l'appropriation du lire et de l'écrire, cet outil se révèle pertinent pour revisiter ces apprentissages avec des publics diversifiés (élèves de l'enseignement adapté, adolescents en grande rupture scolaire et sociale...).

Mots-clés: Appropriation de l'écrit - Grande difficulté scolaire - Langagiciels - Lecture - Langage - Médiation/ remédiation - Outil pédagogique - Outil informatisé - Pédagogie différenciée.

\section{Les Langagiciels: a certain idea of learning behind the software}

Summary: Les Langagiciels is a pedagogical tool that was developed in a computer environment by a group of teachers, trainers, and researchers who were concerned with reading and writing learning difficulties. The article endeavors to highlight the primary characteristics of the tool and its methods of use. It is comprised of seven computer programs, each of which has a priority target of a small number of skills necessary for the acquisition of reading and learning skills. This tool proves to be useful for the revisiting of the acquisition of skills with various target audiences (pupils in adapted instruction, adolescents with major school and social problems, etc.).

Key words: Appropriation of the written word - Computerized tool - Differentiated pedagogy - Langagiciels Language use - Mediation/remediation - Pedagogical tool - Reading - Serious learning difficulties.

$\mathbf{L}$

'OBJET de cet article est de porter à la connaissance du lecteur, un outil pédagogique peu ou mal connu, Les Langagiciels, capable de réconcilier élèves et enseignants avec le plaisir d'apprendre et de faire apprendre, en particulier dans le domaine de la lecture et de l'écriture. Nous présenterons cet outil le plus explicitement possible et nous évoquerons brièvement ses exigences et ses limites, en reprenant ici ou là des éléments existant dans la documentation technique afférente.

Dans le contexte du présent dossier, "L'apport des aides techniques à l'accessibilité à l'école ", nous souhaitons attirer l'attention sur le fait que Les Langagiciels 
ne représentent pas à proprement parler une aide technique: ils constituent en réalité, de notre point de vue, un outil pédagogique à part entière, inséparable d'une démarche et utilisant un environnement informatique. De plus, bien que la question de leur accessibilité à des élèves en situation de handicap soit à l'étude, cet outil relève plutôt pour le moment, d'une utilisation dans le champ de la grande difficulté scolaire.

Sa conception repose sur I'hypothèse suivante: il est possible de mettre en œuvre les remédiations cognitives en travaillant directement dans les champs disciplinaires. Dans ce cadre, le rôle des interactions (apprenants-apprenants, médiateur-apprenants) est essentiel et les activités proposées ambitieuses, tant du point de vue de la complexité des tâches à résoudre que des contenus culturels. Livré avec une bibliothèque de situations de travail prêtes à l'emploi, cet environnement informatique est ouvert. L'enseignant a donc la possibilité de créer lui-même un nombre quasiment illimité de situations sous des formes toujours ajustables, en fonction des objectifs pédagogiques poursuivis et des compétences particulières des apprenants. La diversification des situations de travail, I'adaptation des scénarios au public visé favorise ainsi la mise en œuvre d'une pédagogie différenciée.

Outil de remédiation centré sur une appropriation du lire et de l'écrire, Les Langagiciels traitent de la question du langage dans le champ du français et des langues ainsi que dans le domaine des mathématiques.

C'est un outil particulièrement destiné aux adolescents et aux adultes en grande difficulté d'apprentissage (élèves de Segpa ou d'Erea, adolescents en dispositifs relais, mineurs en CEF (Centres éducatifs fermés), jeunes ou adultes en prison, etc.).

Il peut aussi être utilisé dans le cadre des apprentissages initiaux. Cela suppose quelques pré-requis: concernant les compétences en lecture, le seuil minimal d'accès à l'écrit doit être le mot; par rapport à l'outil informatique, il s'agit de connaître au moins les touches du clavier de l'ordinateur.

Les Langagiciels sont développés par une équipe pluridisciplinaire exerçant dans le champ de la formation initiale et continue, I'association ÉcLire. Ses membres (enseignants, formateurs, chercheurs), ont à cœur l'élaboration d'activités pédagogiques adaptées dans le domaine des apprentissages tardifs en lecture, écriture et mathématiques.

Parce que le cadre associatif offre une certaine indépendance, l'élaboration de cet outil repose sur des opinions librement développées, des partis pris pédagogiques assumés par l'association. Des critiques peuvent lui être légitimement adressées, elles sont recevables cependant dans le cadre des prises de positions affirmées. De plus, ajoutons que les aspects techniques informatiques sont véritablement au service des options pédagogiques et non le contraire.

Pour la petite histoire, c'est en 1981 que les premières formes des Langagiciels ont vu le jour, (sur Micral 8022 G, lorsque les PC n'existaient pas encore). Depuis, le conseil de recherche de l'association ÉcLire a assuré leur développement dans les divers environnements successivement apparus (DOS, puis les différentes versions de Windows). Et en 2002, cet outil a été Reconnu d'intérêt pédagogique (label RIP) par le ministère de l'Éducation nationale. 
À ce jour, Les Langagiciels constituent un ensemble de sept programmes informatiques ${ }^{1}$.

Avant d'aller plus loin, nous voudrions rappeler ce que disait Dominique Barataud ${ }^{2}$ à propos de l'usage d'un outil informatique: "Qu'apporte l'outil présenté qui ne soit pas jouable sans lui ? Si la réponse est: "Rien ", alors, à notre sens, il est à rejeter. Car les contraintes d'utilisation de l'outil informatique sont grandes, en termes de coûts, de conditions d'accompagnement avec ses implications organisationnelles... Si la réponse est positive, reste évidemment à se demander si ce "plus " est intéressant ${ }^{3}$. "

De fait, et concernant la dernière partie de l'affirmation, les concepteurs ont le souci constant de proposer des situations où le support informatique constitue une réelle "plus-value ", d'où l'existence de processus dynamiques dans la mise en œuvre des activités. Si certaines situations de travail peuvent être (et l'ont été par le passé) mises en œuvre en dehors du contexte informatique, la plupart ne sont pas une simple transposition à l'écran de ce qui se ferait avec du papier et un crayon.

Dans le champ du Français, cinq programmes (dits satellites) Rédigeur, Nuages, Tirécrit, Cesécrit, Corécrit, visent chacun l'émergence et le développement de compétences essentielles à la maîtrise du Lire et de l'Écrire. Toutes les activités proposées couplent en permanence ces deux actes; en effet, même si les compétences en lecture et écriture ne sont pas tout à fait les mêmes, nous pensons qu'une pédagogie cohérente d'appropriation du langage ne peut dissocier les deux. De plus, la totalité des compétences nécessaires à la lecture (ou à l'écriture) ne peut être travaillée dans une même séance: ainsi, chaque situation cible prioritairement un nombre limité de compétences.

Relativement à la question de la lecture et de l'écriture, nous pensons que ce qui fait sens pour un apprenant, c'est le texte (unité de sens fondamentale): c'est pourquoi les situations de travail livrées "clés en main " dans l'environnement, ont pour support de vrais textes, des textes forts et ce, quels que soient le niveau de compétence et le niveau de performance des publics avec lesquels on travaille.

Voyons d'abord, le Rédigeur. Cet outil central aux multiples fonctions, sert, en particulier, à produire de l'écrit, à enrichir un écrit, à questionner la nature et la structure d'un texte... et ce, dans des situations voisines de celles qu'on connaît avec un papier et un crayon.

Ainsi il peut se prêter à des activités du genre "Atelier d'écriture " ou "Puzzle ", directement sur l'ordinateur... Notons qu'il n'est pas un traitement de texte de plus dans la panoplie informatique: si nous devons "traiter du texte", nous aurons alors recours aux traitements de texte connus et dont l'usage nous est plus familier.

Autre programme, Nuages est un ensemble de cinq modes de travail liés aux processus visuels engagés dans la lecture (Motamot, Occultation, Ascendant, Descendant, Périodique) dont les objectifs sont de développer les capacités de

1. Un $8^{e}$ programme est en cours de développement qui traitera du problème de la ponctuation.

2. Formateur dans le domaine des enseignements adaptés, D. Barataud est un des co-concepteurs de I'outil Langagiciels.

3. D. Barataud, "Les Langagiciels au service de l'apprenant dans sa conquête de l'écrit ", Les Cahiers de Beaumont, n 64, septembre 1994 (article réactualisé en octobre 2005). 
discrimination et d'anticipation, en confrontant les hypothèses faites sur le sens aux contraintes du code de la langue écrite; pour chacun de ces modes, la nature de l'activité consiste à reconstituer un texte apparaissant progressivement à l'écran et de façon spécifique selon le mode de travail retenu.

Un satellite tout particulier, Tirécrit, propose une activité de reconstitution d'un texte, selon diverses modalités, à partir de sa silhouette (diverses formes sont possibles) et d'indices (lettres, groupes de lettres, etc.) librement déterminés. De nombreuses compétences sont sollicitées: développer des capacités d'exploration systématique; freiner son impulsivité; circuler au sein d'un texte pour s'en approprier le sens; établir des rapports entre structure et sens; mettre en relation phénomènes locaux et articulations textuelles; repérer et intérioriser les marqueurs fondamentaux du code écrit de la langue.

Avec Césécrit, l'apprenant va devoir reconstituer la segmentation en mots d'un texte. L'écran est partagé en deux zones: une zone basse qui est le lieu de travail de l'apprenant, où les écrits apparaissent privées de tout séparateur (espace, apostrophe ou trait d'union); une zone haute au sein de laquelle le texte se réaffiche, au fur et à mesure que les chaînes travaillées en zone basse auront été segmentées correctement. Quatre niveaux de travail, en permanence disponibles, sont proposés, correspondant à une gradation des aides possibles.

Enfin, dernier programme dans le champ du français, Corécrit permet de travailler la dimension orthographique de l'écrit. II s'agit de favoriser chez l'apprenant une prise de conscience de ses propres compétences orthographiques, de l'amener à stabiliser un système minimum de certitudes qui puisse servir de support à de nouveaux apprentissages.

Découpé en blocs (dont la longueur est librement déterminée par l'enseignant), le texte porteur d'erreurs apparaît en zone haute de l'écran. Le travail de modification s'opère en zone basse où chaque bloc peut être isolé. À tout instant l'apprenant peut accéder à une évaluation quantitative et à des aides qualitatives variées et complémentaires.

II est important de faire travailler l'apprenant sur ses propres productions écrites. II s'agira alors d'adapter au programme Corécrit le texte de l'élève avec ses erreurs orthographiques et ainsi de lui permettre de corriger lui-même son écrit.

En résumé, deux grandes familles d'activités peuvent être considérées: celles s'appuyant sur la donnée d'indices stables (Césécrit, Tirécrit, Corécrit et dans Nuages: Occultation); celles mettant en jeu, en plus, des processus dynamiques d'affichage des indices fournis (dans Nuages: Ascendant, Descendant, Mot à Mot, Périodique). Ajoutons que les satellites décrits ci-dessus dans la rubrique "français " permettent en réalité de travailler la question du langage dans d'autres langues (anglais, espagnol, italien, portugais, allemand, etc.): techniquement, les caractères des langues vivantes étrangères sont disponibles dans l'environnement et l'enseignant peut adapter n'importe quel texte dans chacun de ces satellites.

Ces satellites systématisent, dans des modalités de travail différentes, la découverte/le dévoilement d'un écrit caché. II nous semble que cette entrée dans le texte, si difficile à obtenir la plupart du temps, se fait ici plus aisément: elle produit d'ailleurs une appétence 
de la part de l'apprenant qui facilite, entre autres, le travail classiquement attendu en français autour des écrits. Au cours de journées de formation consacrées à un retour sur ces outils, nombreux sont les enseignants ou les éducateurs utilisant régulièrement I'outil, qui témoignent de l'effet d'appropriation des écrits chez l'élève. Une monitrice des Maisons familiales rurales racontait comment, une fois le dévoilement d'un écrit achevé, un élève était impatient de pouvoir imprimer " son " texte et comment elle dut lui faire remarquer qu'il s'agissait d'abord d'un texte de... Victor Hugo!

Dans le champ des mathématiques, le dispositif est complété par deux programmes, Rédigéo et Algécrit, visant des objectifs analogues d'appropriation du langage.

Relativement aux questions d'arithmétique et d'algèbre, Algécrit permet de produire des expressions numériques ou/et algébriques équivalentes à une expression modèle librement déterminée. Les nombres décimaux, les fractions et les puissances peuvent être utilisés et s'affichent comme l'écriture manuscrite. L'objectif visé pour l'apprenant est la prise de conscience et l'appropriation des règles fondamentales des écritures opératoires arithmétiques ou/et algébriques: associativité, commutativité, distributivité de la multiplication par rapport à l'addition, priorités opératoires, écritures opératoires implicites, etc.

Quant à Rédigéo, il vise l'assimilation du langage en géométrie. L'hypothèse faite est que le passage par le langage est incontournable de l'activité de construction en géométrie. Rédigéo travaille donc sur le discours nécessaire à la construction géométrique. II est à noter que ce n'est pas un logiciel de démonstration. Mais comme celle-ci nécessite la maîtrise de la construction et du langage qui l'accompagne, Rédigéo prépare le terrain pour la démonstration en géométrie.

Articulant l'analyse d'un dessin géométrique sur le papier et le discours produit pour construire, il met en évidence si "ce que j'ai écrit énonce bien ce que je voulais dire ". L'apprenant élabore le discours et l'ordinateur construit la figure géométrique correspondante. Nous sommes bien là dans une situation où l'élève est exonéré temporairement de la mise en œuvre d'une procédure (ici, la construction d'une figure) afin de mettre en œuvre une compétence de plus haut niveau (l'identification et la planification des étapes d'une construction).

II nous paraît important de dire quelques mots du contexte nécessaire à l'utilisation de cet outil. Les concepteurs des Langagiciels pensent qu'une réelle pédagogie d'appropriation de l'écrit ne peut exister dans des situations d'individualisation: l'outil est donc élaboré pour des groupes et la résolution d'une tâche sur l'ordinateur prend tout son sens en binôme. Ceci suppose une articulation très forte entre les phases de travail individuel ou par deux et les temps d'échanges, de réflexion et de communication dans le groupe. Ainsi, Les Langagiciels peuvent constituer un support certain pour une véritable pédagogie de médiation et de remédiation. Dans ce cadre, le rôle de l'enseignant est sensiblement modifié. Comme le souligne Philippe Lestievent ${ }^{4}$, "passant d'un registre traditionaliste de transmetteur de

4. P. Lestievent, "Les Langagiciels. La qualité sort de la souche des écrans ", Les Cahiers de Beaumont, n64, septembre 1994 (article réactualisé en octobre 2005). 
connaissances à celui de médiateur à l'élaboration personnelle des savoirs et savoir faire, il se trouve au cœur d'une relation privilégiée avec l'apprenant ".

Cependant, c'est un outil exigeant pour l'enseignant et ce, à différents moments: avant la séance, au moment de la conception d'une situation (une utilisation régulière facilite à long terme cette phase de préparation assez " chronophage »); pendant la séance, où l'enseignant est garant de la démarche de médiation/(re)médiation, tout à la fois organisateur du travail collectif, passeur soucieux de laisser les élèves fonctionner en toute autonomie sur l'ordinateur et, en même temps, personneressource en cas de difficulté... En somme ni trop loin, ni trop proche!

Néanmoins, deux caractéristiques rendent cet outil attrayant de notre point de vue, surtout dans le cadre des enseignements adaptés: d'une part, l'environnement est ouvert et permet de fabriquer ses propres situations pédagogiques; d'autre part, les satellites offrent une réelle possibilité de différencier les situations pédagogiques. Une appropriation complète et pertinente du dispositif nécessite plusieurs jours. De notre point de vue, celle-ci est facilitée dans le cadre de journées de formation ${ }^{5}$. À défaut, quelques articles peuvent être utilement consultés: ceux de Dominique Barataud et de Philippe Lestievent (déjà cités) ainsi que le texte "Regards croisés" qui regroupe les réflexions de quatre auteurs, dont deux professeurs de français en classes relais. Cet article est disponible sur le site Eduscol ${ }^{6}$ du ministère de l'Éducation nationale.

Reste qu'il est bien difficile de rendre compte dans un écrit de l'impact des visualisations dynamiques qui sont au cœur de l'environnement Langagiciels. Comment en effet, sur le papier, rendre sensible ce qui, dans tous les cas, est de l'ordre du mouvement, ce qui relève d'un processus dynamique? Peut-être pouvons-nous alors conseiller de se référer aux films réalisés dans des classes et montrant des séances pédagogiques, certaines ayant pour support Tirécrit, d'autres Rédigéo ${ }^{7}$. Cependant, il n'est pas sûr que la compréhension de l'outil en soit éclairée...

Lire et écrire, activités dont nous avons perdu de vue quelles extraordinaires capacités d'abstraction elles exigent, nécessitent un apprentissage de longue haleine et le temps de l'école n'y suffit pas. Celle-ci crée juste les conditions pour en faciliter l'accès, leur maîtrise ultérieure reste souvent l'affaire de l'apprenant. Nous savons tous en effet, que "plus nous lisons, mieux nous lisons " et "plus nous écrivons, plus nous savons écrire ". Tout le problème réside alors dans l'envie d'aller plus loin, présente ou non, chez l'apprenant.

Cependant, nous reprendrons ici la conclusion d'un des deux articles cités plus haut: "On connaît l'aphorisme de Célestin Freinet: "On ne peut pas faire boire un cheval qui n'a pas soif. "II nous semble aujourd'hui que la vraie question n'est pas

5. Tous les ans, I'INS HEA propose une semaine de formation à cet outil, intitulée: " Langagiciels, structuration du langage, structuration de la pensée, un outil informatique interactif pour la remédiation des difficultés "; voir le plan de formation continue de I'INS HEA: http://www.inshea.fr/FormationsHome.htm

6. Suivre: sommaire - collège - dispositifs relais - outils pédagogiques - "Lire, écrire, un plaisir retrouvé " ; dans le paragraphe "Des pratiques pédagogiques... ", "Regards croisés " fait partie des documents de présentation des films vidéo.

7. Ces films font partie de la collection Utopia-Egpa et leur visionnement nécessite l'examen de leurs documents d'accompagnement. 
tant celle d'une soif éventuelle des apprenants, soif qui, même dans les situations les plus apparemment désespérées (adolescents en rupture scolaire ou/et sociale massive: classes relais, centres éducatifs fermés, quartier mineurs des maisons d'arrêt) nous est toujours apparue très forte, mais celle du caractère au minimum potable et si possible ayant un goût agréable de l'eau présentée. "

Dès lors, la maîtrise du lire et de l'écrire peut être judicieusement approchée par le biais d'activités spécifiques que l'outil ordinateur permet de mettre en chantier et il nous semble que c'est ce que permet l'outil Langagiciels. Nous avons la conviction qu'il favorise une réelle mobilisation cognitive et une authentique activité mentale. Pour s'en convaincre, il suffit d'observer les élèves dans les séances filmées évoquées plus haut, pour repérer une réelle implication de leur part dans l'activité et un véritable travail de réflexion. Bien sûr, une conviction ne suffit pas et il serait utile de mener des études détaillées sur le sujet, afin de transformer une conviction en une argumentation basée sur des analyses plus approfondies. 